\title{
A Feature Fusion Approach for Hand Tools Classification
}

\author{
Mostafa Ibrahim, Alaa Ahmed \\ College of Computer and Information Sciences \\ Al Imam Mohammad Ibn Saud Islamic University (IMSIU) \\ Riyadh, Kingdom of Saudi Arabia
}

\begin{abstract}
The most important functions in objects classification and recognition system are to segment the objects from the input image, extract common features from the objects, and classify these objects as a member of one of the considered object classes. In this paper, we present a new approach for feature-based objects classification. The main idea of the new approach is the fusion of two different feature vectors that are calculated using Fourier descriptors and moment invariants. The fused moment-Fourier feature vector is invariant to image scaling, rotation, and translation. The fused feature vector for a reference object is used for training feed-forward neural network classifier. Classification of some hand tools is used to evaluate the performance of the proposed classification approach. The results show an appreciable increase in the classification accuracy rate with a considerable decrease in the classifier learning time.
\end{abstract}

Keywords-Feature fusion; neural network classifier; invariant features; objects classification

\section{INTRODUCTION}

Pattern recognition is a complicated process that consists of many steps, such as image preprocessing, segmentation, feature extraction and patterns classification. The major steps of the pattern recognition process are the feature extraction and patterns classification. Selection of a feature extraction method is probably the single most important factor in achieving high recognition performance [1].

A principal characteristic of pattern recognition problem is that the number of different objects that the system has to cope with is unlimited. That is mainly because of the various shapes of the object, e.g., by varying object scale, orientation and translation. Thus, the problem can't be solved by straightforward template matching. Each object must be classified to one of the pattern classes. The Neural Network (NN) classifier is supervised trained based on a sample of typical objects representing different classes. The classifier then can classify new unknown objects with minimum error.

In the presented paper, we introduce a feature fusion based approach for pattern classification. The proposed fused feature vector consists of reduced Fourier descriptors and four moment invariants. The new approach aims to enhance the classification rate and learning time of the neural network classifier by taking advantage of the inherent complementary of the Fourier descriptors and moment invariants features.

The rest of the paper is organized as follows: In Section 2, the related work is introduced. The proposed work is presented in Section 3. Section 4 presents the pattern classifier. Section 5 shows the experimental results. Finally, the paper ends with the conclusion.

\section{RELATED WORK}

In literature, there are many image feature extraction methods that have been offered by researchers. These approaches can be categorized into either holistic or local image feature extraction. The first is based on producing statistical information pattern from a big number of training samples of data such as the principal component analysis (PCA) [2]. PCA is improved by many researchers such as 2D PCA [3], block PCA [4], or incremental PCA [5]. Also, matrix decomposition and linear combination are heavily used methods [6]. The holistic approaches have a couple of disadvantages. They don't take into consideration the local detailed information. They are easily affected by the geometric shape changes and noise variation. The local feature extraction methods can prevail over these disadvantages. In [7] authors presented a texture feature description method. It is called a local binary pattern method (LBP). The method is based on computing a binary sequence with $3 \times 3$ neighborhoods matrix. The central pixel values are compared to its neighbors and then an LBP histogram is produced as a texture description feature.

The fixed neighborhoods make it easy to restrict the larger neighborhood structure, which is an obvious disadvantage for the LBP method. In [8], [9] authors introduced a region based method with a co-occurrence matrix. This method gives good results especially with medical data sets. To overcome the illumination sensitivity problem, Gabor wavelets filters are considered as a good candidate that could be used in handling this issue. It is an excellent feature representation that is not affected by the illumination or expression variation. In literature, there are a lot of Gabor Extraction methods which give very outstanding results with good performance indicator and ability to work with wide range of applications such as local normalization entropy-like weighted Gabor features [10], Gabor wavelets combined with volumetric fractal dimension [11] and fusion of multi-channels classifier [12]. Neural networks have been extensively used for purpose of objects classification. Among several NN architectures the BackPropagation model is the most widely used [13]. In [14] authors presented an Extension Neural Network (ENN) for recognizing the tool cutting state. Their results show shorter learning time and better recognition accuracy. 


\section{PROPOSED APPROACH}

Fig. 1 illustrates the proposed objects classification process using the feature fusion approach.

A gray scale images are acquired for each object by a digital camera. These gray scale images are then converted to binary images. An object pixel is considered a boundary point if any of its neighbors is classified as background pixel. Tracking the boundary around a pattern is the process of keeping only those pixels that form the boundary of that pattern. This process transforms the two-dimensional binary image into a scalar vector representing the coordinates of boundary points of that pattern. Each boundary is uniquely determined by specifying the location of its initial point and a sequence of points having the same labels as the initial point. Contour tracking have been done using Left Most Looking (LML) algorithm [15]. By taking the Discrete Fourier Transform (DFT) of the object contours, we obtained 16 Fourier descriptors feature vector, which have been handled to become rotation, scale and translation invariant, the same way as described in [16].

A reduced Fourier descriptor feature vector of only 8 descriptors has been computed, by Appling the DFT on the ordinary 16 Fourier descriptors feature vector [17].

Hu's seven moment invariants that are invariant under translation, rotation, and scaling have been calculated from the objects [15], [18]-[20].

$$
\begin{aligned}
& \phi_{1}=\eta_{20}+\eta_{02}, \\
& \phi_{2}=\left(\eta_{20}-\eta_{02}\right)^{2}+4 \eta_{11}^{2}, \\
& \phi_{3}=\left(\eta_{30}-3 \eta_{12}\right)^{2}+\left(3 \eta_{21}-\eta_{03}\right)^{2}, \\
& \phi_{4}=\left(\eta_{30}+\eta_{12}\right)^{2}+\left(\eta_{21}+\eta_{03}\right)^{2}, \\
& \varphi_{5}=\left(\eta_{30}-3 \eta_{12}\right)\left(\eta_{30}+\eta_{12}\right)\left(\left(\eta_{30}+\eta_{12}\right)^{2}-3\left(n_{21}+n_{03}\right)^{2}+\left(3 n_{21}-n_{03}\right)\right) \\
& \left(\eta_{21}+\eta_{03}\right)\left(3\left(\eta_{30}+\eta_{12}\right)^{2}-\left(\eta_{21}+\eta_{03}\right)^{2}\right), \\
& \varphi_{6}=\left(\eta_{20}-\eta_{02}\right)\left(\left(\eta_{30}+\eta_{12}\right)^{2}-\left(\eta_{21}+\eta_{03}\right)^{2}\right)+4 \eta_{11}\left(\eta_{30}+\eta_{12}\right)\left(\eta_{21}+\eta_{03}\right), \\
& \varphi_{7}=\left(3 \eta_{21}-\eta_{03}\right)\left(\eta_{30}+\eta_{12}\right)\left(\left[\eta_{30}+\eta_{12}\right)^{2}-3\left(\eta_{21}+\eta_{03}\right)^{2}\right]+\left(3 \eta_{12}-\eta_{30}\right)\left(\eta_{21}+\eta_{03}\right) \\
& X\left(3\left(\eta_{30}+\eta_{12}\right)^{2}-\left(\eta_{21}+\eta_{03}\right)^{2}\right),
\end{aligned}
$$

Where, $\eta_{p q}$ is the normalized central moment of order $(p+q)$

$$
\eta_{p q}=\frac{\mu_{p q}}{\mu_{\mathrm{oO}}^{w}}, w=\frac{p+q}{2}+1
$$

and $\mu_{\mathrm{pq}}$ is the central moment of order $(\mathrm{p}+\mathrm{q})$. For binary pattern $\mathrm{G}$ is defined as

$$
\mu_{p q}=\sum \sum_{G}\left(x-x_{c}\right)^{p}\left(y-y_{c}\right)^{q}
$$

Where, $\left(\mathrm{x}_{\mathrm{c}}, \mathrm{y}_{\mathrm{c}}\right)$ are coordinates of the center of gravity of pattern $\mathrm{G}$ and are defined as:

$$
x_{c}=\frac{m_{10}}{m_{\mathrm{OO}}}, \quad y_{c}=\frac{m_{\mathrm{O} 1}}{m_{\mathrm{OO}}}
$$

Where, $\mathrm{m}_{\mathrm{pq}}$ is the regular geometric moment and for binary pattern $\mathrm{G}$ defined as:

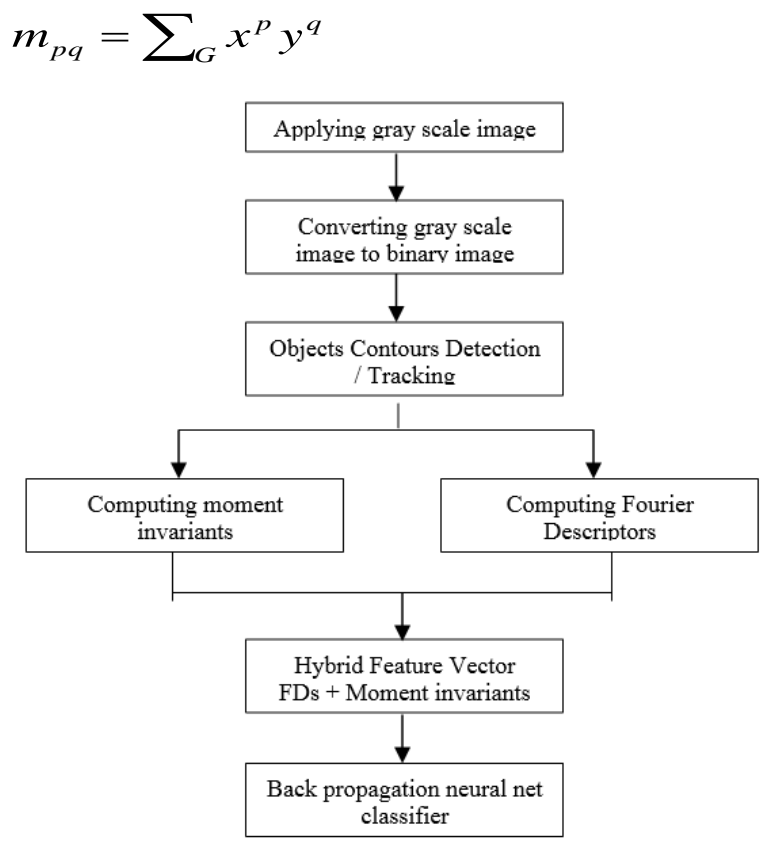

Fig. 1. Proposed objects classification method.

Aiming to enhance the classification accuracy rate and reducing the training time, we developed a new approach for objects classification by combining the moment invariants feature vector with the reduced Fourier descriptors feature vector. Reduced Fourier descriptors are calculated from the object contours while moment invariants are calculated from the objects body. Thus, the fused feature vector is developed to achieve higher classification accuracy rate and reliability by taking advantage of the inherent complementary of the two feature extraction methods. We have used the first four moment invariants $(\phi 1, \phi 2, \phi 3$ and $\phi 4)$ in addition to the eight reduced Fourier descriptors resulting in a fused feature vector of 12 coefficients.

\section{Neural Network Classifier}

The Feed-forward neural network is the most frequently used among many neural network paradigms, which satisfy the requirements of pattern classification [18], [21]. A Feedforward neural network, consisting of an input layer, a hidden layer, and an output layer, is considered as shown in Fig. 2. The input layer has 8, 7 and 12 nodes in case of reduced Fourier descriptors, moment invariants and fused feature vectors respectively. The hidden layer has 6,4 and 8 nodes in case of reduced Fourier descriptors, moment invariants and fused feature vectors, respectively. While the output layer has only three nodes. Each processing element (neuron) is connected to all those in the adjacent layers. The Sigmoid function is used as node transfer function. 


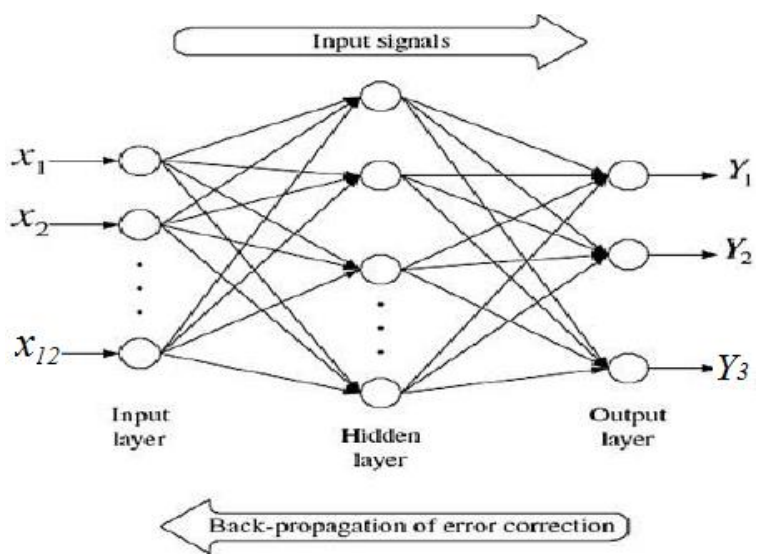

Fig. 2. Neural network classifier using proposed fused feature vector.

We have considered four kinds of hand tools as training patterns; so three output nodes are sufficient as they can represent up to eight different patterns. The output should be (001) for pattern number 1, (010) for pattern number 2, (011) for number 3 and so on. For the neural network training, we have applied "back-propagation learning algorithm" with momentum. All the weights are initially set to random values with a uniform distribution over $[-0.5,0.5]$. The patterns in Fig. 3 have been used as training patterns. The training error in case of using any of the feature vectors found to be $<0.0001$ with learning rate of 0.5 and momentum term of 0.7 .

For classifying of the test patterns, we have defined an output vector \{out (1), out (2), and out (3) \} by thresholding neural net outputs zi in such a way that for $i=1,2,3$

$$
\text { out }(i)=\left\{\begin{array}{cc}
1 & \text { for } \mathrm{zi} \geq 0.9 \\
0 & \text { for zi } \leq 0.1 \\
\text { unknown } & \text { for } 0.1<\mathrm{z}_{i}<0.9
\end{array}\right.
$$

\section{RESULTS}

This section represents the conducted experimentations along with their results. Two experiments have been conducted. The first is to recognize hand tools. While the second experiment is to recognize rotated and scaled version of these hand tools.

The first experiment is to classify some hand tools without any variations in scale, rotation or translation. As a sample for this experiment four objects have been assigned as shown in Fig. 3(a). First, the objects contours have been detected and tracked as shown in Fig. 3(b). Second, the ordinary Fourier Descriptors (FDs) and the reduced FDs feature vectors have been calculated as indicated in Table 1 . Then, we calculated the seven moment invariants and the hybrid feature vector as shown in Tables 2 and 3, respectively. Finally, the feature vectors that were extracted using the above-mentioned methods have been applied to a back propagation neural network classifier.
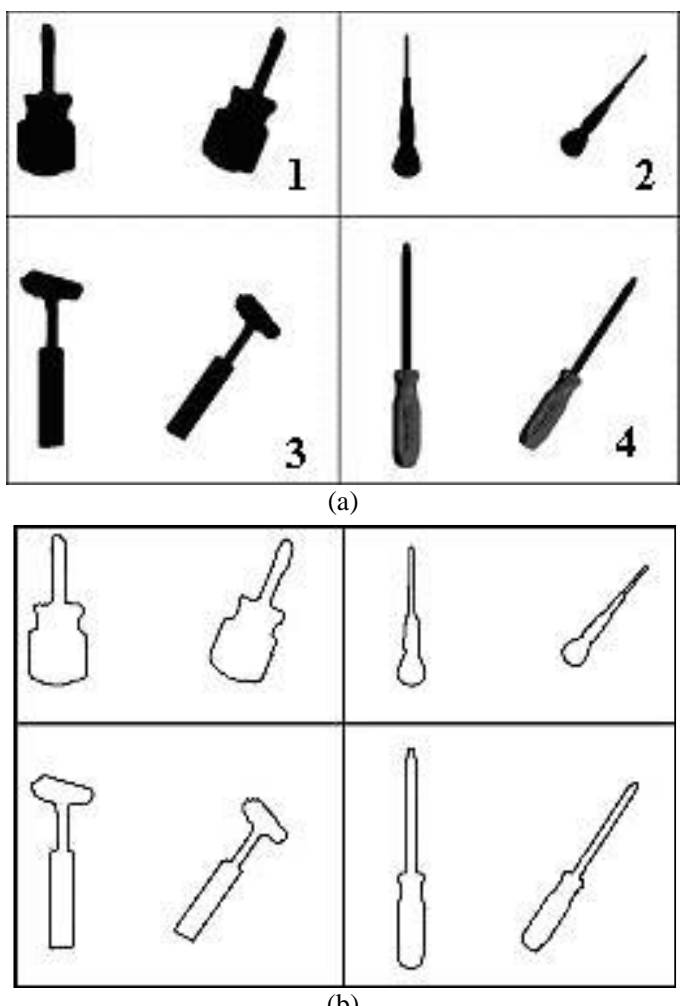

Fig. 3. Training set of objects, 2 samples per object. (a) Training objects. (b) Training object's contours.

TABLE I. REDUCED FOURIER DESCRIPTORS FEATURE VECTORS

\begin{tabular}{|l|l|l|l|l|l|l|l|l|}
\hline & \multicolumn{2}{|l|}{ Object-1 } & \multicolumn{2}{l|}{ Object-2 } & \multicolumn{2}{l|}{ Object-3 } & \multicolumn{2}{l|}{ Object-4 } \\
\hline F1 & 0.310 & 0.311 & 0.240 & 0.229 & 0.358 & 0.309 & 0.198 & 0.186 \\
\hline F2 & 0.276 & 0.282 & 0.154 & 0.145 & 0.270 & 0.242 & 0.154 & 0.140 \\
\hline F3 & 0.277 & 0.279 & 0.175 & 0.167 & 0.295 & 0.225 & 0.203 & 0.182 \\
\hline F4 & 0.330 & 0.339 & 0.170 & 0.177 & 0.300 & 0.231 & 0.241 & 0.215 \\
\hline F5 & 0.033 & 0.048 & 0.027 & 0.012 & 0.035 & 0.077 & 0.063 & 0.056 \\
\hline F6 & 0.198 & 0.188 & 0.074 & 0.097 & 0.128 & 0.153 & 0.104 & 0.103 \\
\hline F7 & 0.460 & 0.456 & 0.178 & 0.207 & 0.349 & 0.294 & 0.204 & 0.183 \\
\hline F8 & 0.255 & 0.251 & 0.102 & 0.120 & 0.218 & 0.172 & 0.123 & 0.124 \\
\hline
\end{tabular}

TABLE II. MOMENT INVARIANT FEATURE VeCTORS

\begin{tabular}{|l|l|l|l|l|l|l|l|l|}
\hline & \multicolumn{2}{|l|}{ Object-1 } & \multicolumn{2}{l|}{ Object-2 } & \multicolumn{2}{l|}{ Object-3 } & \multicolumn{2}{l|}{ Object-4 } \\
\hline M1 & 0.296 & 0.296 & 0.758 & 0.780 & 0.712 & 0.716 & 0.978 & 0.968 \\
\hline M2 & 0.047 & 0.048 & 0.524 & 0.560 & 0.437 & 0.443 & 0.914 & 0.895 \\
\hline M3 & 0.016 & 0.016 & 0.280 & 0.269 & 0.002 & 0.003 & 0.364 & 0.375 \\
\hline M4 & 0.009 & 0.009 & 0.242 & 0.235 & 0.000 & 0.000 & 0.337 & 0.328 \\
\hline M5 & 0.000 & 0.000 & 0.063 & 0.059 & 0.000 & 0.000 & 0.118 & 0.115 \\
\hline M6 & 0.002 & 0.002 & 0.175 & 0.176 & 0.000 & 0.000 & 0.322 & 0.310 \\
\hline M7 & 0.010 & 0.010 & 0.012 & 0.012 & 0.017 & 0.017 & 0.011 & 0.010 \\
\hline
\end{tabular}


TABLE III. HYBRID FEATURE VECTORS

\begin{tabular}{|l|l|l|l|l|l|l|l|l|}
\hline & \multicolumn{2}{|l|}{ Object-1 } & \multicolumn{2}{l|}{ Object-2 } & \multicolumn{2}{l|}{ Object-3 } & \multicolumn{2}{l|}{ Object-4 } \\
\hline F1 & 0.542 & 0.541 & 0.357 & 0.354 & 0.552 & 0.514 & 0.318 & 0.315 \\
\hline F2 & 0.310 & 0.311 & 0.240 & 0.229 & 0.358 & 0.309 & 0.198 & 0.186 \\
\hline F3 & 0.276 & 0.282 & 0.154 & 0.145 & 0.270 & 0.242 & 0.154 & 0.140 \\
\hline F4 & 0.277 & 0.279 & 0.175 & 0.167 & 0.295 & 0.225 & 0.203 & 0.182 \\
\hline F5 & 0.330 & 0.339 & 0.170 & 0.177 & 0.300 & 0.231 & 0.241 & 0.215 \\
\hline F6 & 0.033 & 0.048 & 0.027 & 0.012 & 0.035 & 0.077 & 0.063 & 0.056 \\
\hline F7 & 0.198 & 0.188 & 0.074 & 0.097 & 0.128 & 0.153 & 0.104 & 0.103 \\
\hline F8 & 0.460 & 0.456 & 0.178 & 0.207 & 0.349 & 0.294 & 0.204 & 0.183 \\
\hline F9 & 0.296 & 0.296 & 0.758 & 0.780 & 0.712 & 0.716 & 0.978 & 0.968 \\
\hline F10 & 0.047 & 0.048 & 0.524 & 0.560 & 0.437 & 0.443 & 0.914 & 0.895 \\
\hline F11 & 0.016 & 0.016 & 0.280 & 0.269 & 0.002 & 0.003 & 0.364 & 0.375 \\
\hline F12 & 0.009 & 0.009 & 0.242 & 0.235 & 0.000 & 0.000 & 0.337 & 0.328 \\
\hline
\end{tabular}

In this experiment, we have examined our classifier by applying the same feature vectors of the objects in Fig. 3(b) that have been used for training the neural net classifiers. The results showed that the recognition rate is $100 \%$ in all approaches.

The second experiments evaluate the performance of the proposed objects classification technique with rotated, scaled and translated samples of the reference objects. Fig. 4(a) and (b) show scaled, translated and rotated versions of the objects that have been used in training the neural network classifier to be used as testing sets of patterns. While Fig. 4(c) shows the set of hand tools used for testing.

The first stage in this experiment is to obtain the first 16 Fourier descriptors and the reduced FDs for those patterns, then, use the resulted reduced FDs feature vectors as input to the classifier and record the results of recognition process. The second stage is to obtain the seven moment invariants feature vector and the hybrid feature vector for each pattern to use them as input feature vectors for the neural network classifier, then, records the results of recognition. At last, the results of both stage's recognition rates are compared.

For the performance evaluation of the classifier using the feature vectors computed in first and second stages; a large testing set for each of the target objects was constructed to have seven scale variations from 1.0 to 0.4 , for each of 12 rotation angles, a total of 12 rotation angles times 7 scale factors equals 84 testing samples. This results in a testing set of 336 patterns for the four target objects. Table 4 shows a comparison between the recognition rates using the three feature vectors.

TABLE IV. COMPARISON BETWEEN RECOGNITION RATES

\begin{tabular}{|c|c|c||c|}
\hline Object & Reduced FDs & Moments & Fused Feature vector \\
\hline $\mathbf{1}$ & $97.62 \%$ & $100 \%$ & $100 \%$ \\
\hline $\mathbf{2}$ & $92.86 \%$ & $92.86 \%$ & $97.62 \%$ \\
\hline $\mathbf{3}$ & $97.62 \%$ & $100 \%$ & $100 \%$ \\
\hline $\mathbf{4}$ & $92.86 \%$ & $92.86 \%$ & $95.24 \%$ \\
\hline Training time (ms) & 3855 & 2704 & 1673 \\
\hline
\end{tabular}

\begin{tabular}{|l|l|l||c|}
\hline No. of I/P Layer Nodes & 8 & 7 & 12 \\
\hline
\end{tabular}

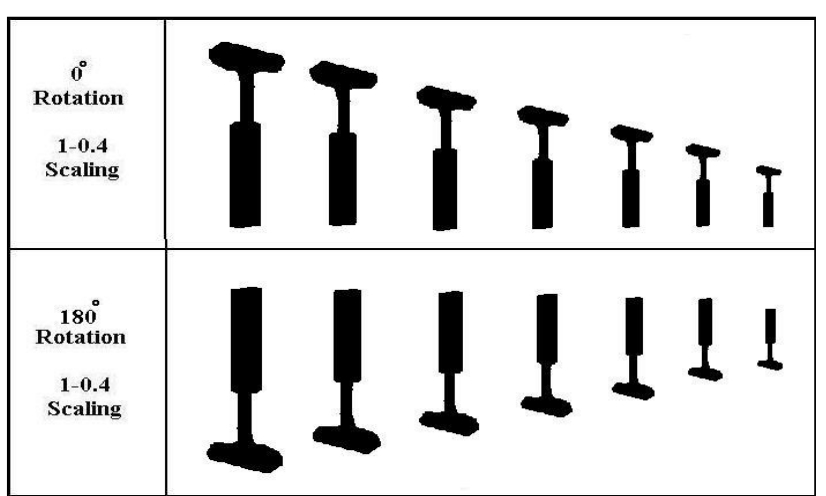

(a)

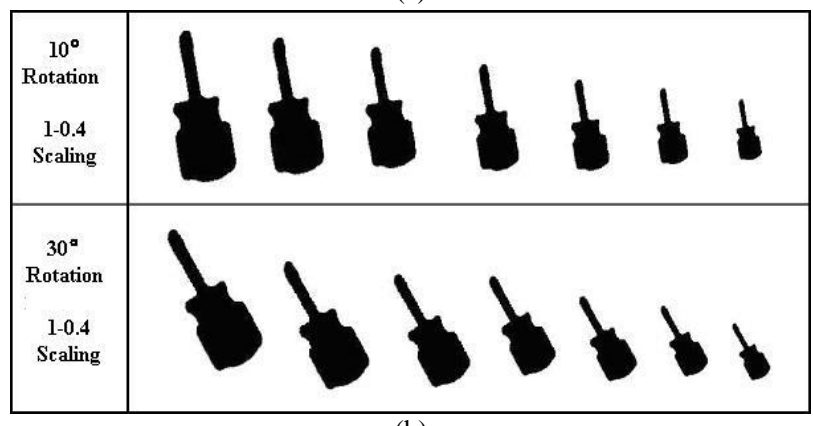

(b)

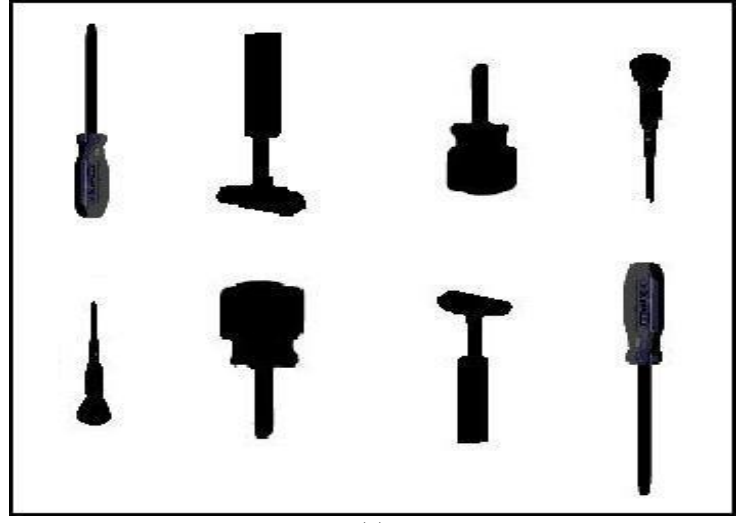

(c)

Fig. 4. Sample of testing objects set.

\section{CONCLUSIONS}

This paper introduces a feature fusion based approach for objects classification. The proposed feature fusion based approach consists of a fused feature vector of four moment invariants and eight reduced Fourier descriptors. The fused feature vector is invariant to object scaling, rotation and translation. A feed-forward neural network has been used as objects classifier. The proposed approach has been applied to recognize and classify four different hand tools. The results show that the use of fused feature vector outperforms the individual use of Fourier descriptors or moment invariants. The results show an appreciable increase in the classification accuracy rate while considerably decrease the classifier learning time compared to the learning time in case of using reduced FDs or moment invariants feature vectors. 


\section{REFERENCES}

[1] J. Miaoa, L. Niub, "A survey on feature selection" Information Technology and Quantitative Management (ITQM 2016), Procedia Computer Science, Vol 91 , 919-926, 2016.

[2] M.A Turk, A.P Pentland, "Face recognition using eigenfaces" In Proceedings of the IEEE Computer Society Conference on Computer Vision and Pattern Recognition, pp. 586-591. 2, 1991 IEEE.

[3] J. Yang, C Liu "Horizontal and vertical 2DPCA-based discriminant analysis for face verification on a large-scale database" IEEE Trans. Inf. Forensics Secur, vol 2, pp 781-792, 2007.

[4] K Tan, S. Chen, "Adaptively weighted sub-pattern PCA for face recognition" Neurocomputing, vol 64, pp 505-511, 2005.

[5] D. Huang, Z. Yi, X, "A new incremental PCA algorithm with application to visual learning and recognition" Neural Process. Lett, vol 30, pp 171-185, 2009.

[6] S. Selvan, P.B Borckmans, A. Chattopadhyay, P.A. Absil, "Spherical mesh adaptive direct search for separating quasi-uncorrelated sources by range-based independent component analysis" Neural Comput, Vol 25, pp. 2486-2522, 2013

[7] T. Ojala, M Pietikäinen, T. Mäenpää, "A generalized local binary pattern operator for multiresolution gray scale and rotation invariant texture classification" In Proceedings of the International Conference on Advances in Pattern Recognition, Volume 1, pp. 397-406, 2001, Brazil.

[8] L. Nanni, S. Brahnam, S. Ghidoni, M. Emanuele, B. Tonya, "Different approaches for extracting information from the co-occurrence matrix" PLoS ONE, vol 8, 2013.

[9] L. Nann, S. Brahnam, S. Ghidoni, E. Menegatti, "Region based approaches and descriptors extracted from the cooccurrence matrix" Int. J. Latest Res. Sci. Technol, vol 3, pp 192-200, 2014.

[10] L.A Cament, L.E. Castillo, J.P. Perez, F.J. Galdames, C.A. Perez, "Fusion of local normalization and Gabor entropy weighted features for face identification" Pattern Recognit. vol 47, pp. 568-577, 2014
[11] A.G.Zuñiga, J.B. Florindo, O.M, Bruno "Gabor wavelets combined with volumetric fractal dimension applied to texture analysis" Pattern REcognit. Lett, Vol 36, pp. 135-143, 2014.

[12] Z.S. Zhao, L. Zhang, M. Zhao, Z.G Hou, C.S Zhang, "Gabor face recognition by multi-channel classifier fusion of supervised kernel manifold learning" Neurocomputing, vol 97, pp. 398-404, 2012

[13] P Nie, X Chen, "Prediction of tool VB value based on PCA and BP neural network" In Journal of Beijing University of Aeronautics and Astronautics, Vol. 37, 3, pp 364-367, 2011

[14] Yu Zhou, Tingling Wang, ENN-based recognition method for tool cutting state, In Journal of Computational Science, 2017, ISSN 18777503, In press Available online 24 July 2017doi: https://doi.org/10.1016/j.jocs.2017.07.014

[15] M-C Roha, T-Y Kima, J. Parkb, S-W. Leea , "Accurate object contour tracking based on boundary edge selection", Pattern Recognition Vol. 40, Issue 3, Pages 931-943, March 2007.

[16] L. Zhang and J. Pu, "An improved back propagation neural network in objects recognition," IEEE International Conference on Automation and Logistics (ICAL), Chongqing, 2011, pp. 507-511, 2012.

[17] E. Sokic, S. Konjicija, "Phase preserving Fourier descriptor for shapebased image retrieval, Signal Processing" Image Communication, vol. 40, pp. 82-96, 2016, Elsevier

[18] Z. Huang, J. Leng “Analysis of Hu's moment invariants on image scaling and rotation" Computer Engineering and Technology (ICCET), pp16-18 April 2010

[19] J. flusser, t. suk and b. zitova, "Moments and moment invariants in pattern recognition", Wiley, 2009

[20] M. Gong, Y. Hao, H. Mo , H. Li,'Naturally combined shape-color moment invariants under affine transformations" Computer Vision and Image Understanding , pp. 1-11, 2017, Elsevier.

[21] D. ArunKumar, S.Meher, Deban. Kanhar and K.PadmaKumari "Unified granular neural networks for pattern classification", Neurocomputing, Vol. 216, 5, pp 109-125, Elsevier, 2016. 